\title{
Distinct Trajectories of Cortisol Response to Prolonged Acute Stress Are Linked to Affective Responses and Hippocampal Gray Matter Volume in Healthy Females
}

\author{
Roee Admon, ${ }^{1 \star}$ Michael T. Treadway, ${ }^{2 \star}$ Linda Valeri, ${ }^{3}{ }^{\circledR}$ Malavika Mehta, ${ }^{4}$ Samuel Douglas, ${ }^{4}$ and $\odot$ Diego A. Pizzagalli4,5 \\ ${ }^{1}$ Department of Psychology, University of Haifa, Haifa, Israel 3498838, ${ }^{2}$ Department of Psychology, Emory University, Atlanta, Georgia 30322, and \\ ${ }^{3}$ Psychiatric Biostatistics Laboratory, ${ }^{4}$ Center for Depression, Anxiety, and Stress Research, and ${ }^{5}$ McLean Imaging Center, McLean Hospital/Harvard \\ Medical School, Belmont, Massachusetts 02478
}

The development of robust laboratory procedures for acute stress induction over the last decades has greatly advanced our understanding of stress responses in humans and their underlying neurobiological mechanisms. Nevertheless, attempts to uncover linear relationships among endocrine, neural, and affective responses to stress have generally yielded inconsistent results. Here, 79 healthy females completed a well established laboratory procedure of acute stress induction that was modified to prolong its effect. Endocrinological and subjective affect assessments revealed stress-induced increases in cortisol release and negative affect that persisted 65 and 100 min after stress onset, respectively, confirming a relatively prolonged acute stress induction. Applying latent class linear mixed modeling on individuals' patterns of cortisol responses identified three distinct trajectories of cortisol response: the hyper-response $(n=10)$, moderate-response $(n=21)$, and mild-response $(n=48)$ groups. Notably, whereas all three groups exhibited a significant stress-induced increase in cortisol release and negative affect, the hyper-response and mild-response groups both reported more negative affect relative to the moderate-response group. Structural MRI revealed no group differences in hippocampal and amygdala volumes, yet a continuous measure of cortisol response (area under the curve) showed that high and low levels of stress-induced cortisol release were associated with less hippocampal gray matter volume compared with moderate cortisol release. Together, these results suggest that distinct trajectories of cortisol response to prolonged acute stress among healthy females may not be captured by conventional linear analyses; instead, quadratic relations may better describe links between cortisol response to stress and affective responses, as well as hippocampal structural variability.

Key words: cortisol; hippocampus; HPA; mood; stress

\section{Significance Statement}

Despite substantial research, it is unclear whether and how individual neuroendocrine stress response patterns are linked to affective responses to stress and structural variability in neuroendocrine regulatory brain regions. By applying latent class linear mixed modeling on individuals' patterns of cortisol responses to a prolonged acute stressor, we identified three distinct trajectories of cortisol response. Relative to the group showing a moderate cortisol response, groups characterized by hyper and mild cortisol response were both associated with more negative affect. Moreover, a continuous measure of cortisol response showed that high and low levels of stress-induced cortisol release correlated with reduced hippocampal gray matter volume. Given that neuroendocrine stress responses are conceptualized as biomarkers of stress susceptibility, these insights may have clinical implications.

\section{Introduction}

Stress sensitivity is a key element in the etiology and pathophysiology of psychopathology (Harkness et al., 2015). Accordingly, extensive scientific effort has been devoted to the characterization of the neural and physiological responses to acute stress that accompany the typical stress-induced temporary shift toward more 
negative affective state. This work established that the physiological response to acute stress involve the activation of endocrine stress-response systems, most prominently the hypothalamuspituitary-adrenal (HPA) axis (Herman et al., 2016). As the end point of the HPA system, salivary cortisol is the most frequently used variable to evaluate endocrine stress response in laboratory settings, with a cortisol increase $\geq 2.5 \mathrm{nmol} / \mathrm{L}$ after stress induction typically taken as a threshold for "stress response" (Foley and Kirschbaum, 2010). A wealth of laboratory acute stress procedures established that $\sim 50-80 \%$ of individuals can be classified as "stress responders" based on this cutoff (Dickerson and Kemeny, 2004). Interestingly, however, in most cases, links between stress-induced cortisol and affective responses are not clear, with the majority of studies not reporting significant differences in affective responses to stress among stress responders compared with nonresponders (Campbell and Ehlert, 2012). Further, only $\sim 25 \%$ of studies report a linear relation between cortisol and affective responses to stress (Campbell and Ehlert, 2012).

The endocrine stress-response systems have also been the focus of neuroimaging research, particularly targeting the hippocampus and amygdala structures because of their pivotal roles in HPA regulation. Results have been mostly inconsistent, with hippocampal volume being positively associated (Pruessner et al., 2007), negatively associated (Cho, 2001), or not associated (Liu et al., 2012) with the magnitude of cortisol response to stress. Similarly mixed results also emerged with regard to amygdala volume (Klimes-Dougan et al., 2014; Cacciaglia et al., 2017). Together, attempts to link patterns of stress-induced cortisol response to both affective responses and structural variability in HPA-regulating brain regions have yielded mixed results. One potential explanation for these inconsistencies may relate to the fact that studies typically report on mean cortisol response or use predetermined values to classify individuals as stress responders versus nonresponders, thus disregarding the important role of individual differences in determining stress sensitivity (Monroe and Simons, 1991; Liu, 2015). In addition, given that current laboratory protocols induce stress for a relatively short time period (typically $<20 \mathrm{~min}$ ), it is possible that induced effects were too brief to allow sufficient endocrine and emotional variability to evolve.

To address these limitations, the first aim of the current study was to induce acute stress effectively for a relatively prolonged time period among healthy females while capturing individual endocrine and affective response patterns. To this end, healthy females completed the Maastricht Acute Stress Test (MAST), a robust laboratory acute stress procedure (Smeets et al., 2012) that was modified to prolong its effect by informing participants upon task completion that, due to their poor performance, they would need to repeat the task later in the session. Our second aim was to identify distinct trajectories of cortisol response to such prolonged acute stress without a priori assumptions regarding the number, size, or pattern of change of these trajectories. This was accomplished by applying latent class linear mixed modeling (LCMM) on individuals' patterns of cortisol responses. Our final aim pertained to investigating in a subsample $(n=69)$ with MRI data potential links between cortisol response to stress and struc-

Correspondence should be addressed to either of the following: Roee Admon, Ph.D., Department of Psychology, University of Haifa, 199 Aba Khoushy Ave., Haifa, Israel 3498838, E-mail: radmon@psy.haifa.ac.il; or Diego A. Pizzagalli, Ph.D., Center for Depression, Anxiety and Stress Research, McLean Hospital/Harvard Medical School, 115 Mill Street, McLean Hospital, Belmont, MA 02478, E-mail: dap@mclean.harvard.edu.

DOI:10.1523/JNEUROSCI.1175-17.2017

Copyright $\odot 2017$ the authors $\quad 0270-6474 / 17 / 377995-09 \$ 15.00 / 0$
Table 1. Sample demographic information

\begin{tabular}{|c|c|}
\hline & $n(\%)$ \\
\hline \multicolumn{2}{|l|}{ Gender } \\
\hline Female & $88(100 \%)$ \\
\hline \multicolumn{2}{|l|}{ Handedness } \\
\hline Right & $88(100 \%)$ \\
\hline \multicolumn{2}{|l|}{ Race } \\
\hline Caucasian & $61(69 \%)$ \\
\hline Black & $15(17 \%)$ \\
\hline Asian & $10(12 \%)$ \\
\hline Unknown & $2(2 \%)$ \\
\hline \multicolumn{2}{|l|}{ Ethnicity } \\
\hline Non-Hispanic & $81(92 \%)$ \\
\hline Hispanic & $5(6 \%)$ \\
\hline Unknown & $2(2 \%)$ \\
\hline \multicolumn{2}{|l|}{ Income } \\
\hline$<\$ 10,000$ & $11(13 \%)$ \\
\hline$\$ 10,000-25,000$ & $10(11 \%)$ \\
\hline$\$ 25,000-50,000$ & $21(24 \%)$ \\
\hline$\$ 50,000-75,000$ & $18(20 \%)$ \\
\hline$\$ 75,000-100,000$ & $17(19 \%)$ \\
\hline$>\$ 100,000$ & $11(13 \%)$ \\
\hline \multicolumn{2}{|l|}{ Marital status } \\
\hline Married & 17 (19\%) \\
\hline Unmarried & $71(81 \%)$ \\
\hline \multicolumn{2}{|l|}{ Smoking status } \\
\hline Nonsmoker & $88(100 \%)$ \\
\hline \multicolumn{2}{|l|}{ Menstrual cycle phase } \\
\hline Follicular & $62(70 \%)$ \\
\hline Luteal & $26(30 \%)$ \\
\hline \multicolumn{2}{|l|}{ Contraceptive usage } \\
\hline Yes & $44(50 \%)$ \\
\hline Education (median, y) & $16.5(1.7)$ \\
\hline BMI (median) & $22.6(3.4)$ \\
\hline
\end{tabular}

tural variability with a priori hypotheses relating to key regions implicated in HPA regulation such as the amygdala and hippocampus. Overall, we hypothesized that applying a data-driven approach on cortisol patterns of response to stress may provide a more accurate account for individual variability and that these insights may enable linking stress-induced cortisol responses with affective responses and with structural variability in regions implicated in HPA regulation.

\section{Materials and Methods}

Participants. A total of 88 right-handed psychiatrically, medically, and neurologically healthy female participants were included. Only females were investigated to avoid potential sex-dependent variability in HPA axis stress response (Kudielka and Kirschbaum, 2005). All participants were recruited using community advertisements. Exclusion criteria included any current or past psychiatric disorder as assessed by a Structured Clinical Interview for the DSM-IV (SCID; First et al., 2005). In addition, individuals were excluded for five or more lifetime exposures to any illegal substance, as well as due to recent use of illegal drugs, psychotropic medications, or nicotine. For a complete summary of relevant demographic characteristics, please see Table 1.

Study procedure. Participants were tested in individual sessions between 11:00 A.M. and 4:00 P.M. to minimize the effects of diurnal variation on endogenous cortisol levels (Blascovich et al., 2011). The stress procedure itself occurred between 1:00 P.M. and 2:00 P.M. Further, to allow for controlled saliva collection, participants were asked not to brush their teeth and to refrain from food, drinks, and intense physical exercise at least $1 \mathrm{~h}$ before the test phase. None of the participants reported to have violated these directives. Upon arrival, participants received information about the study and the measurements that would be taken and provided written informed consent to a protocol approved by 


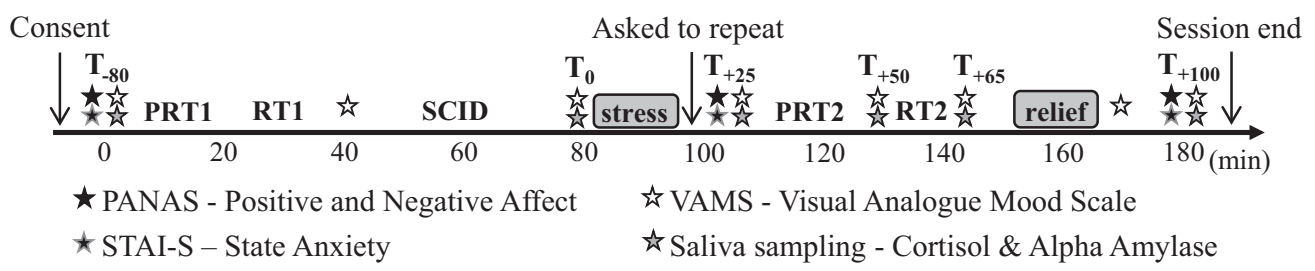

Figure 1. Schematic diagram illustrating the timeline and design of the study session. Participants provided written consent before completing two tasks, one probabilistic reward task (PRT) and one reaction time (RT) task, a clinical interview to determine eligibility (SCID), the MAST, and then the PRT and RT tasks again. To prolong the effect of the acute stressor, immediately upon MAST completion, participants were told by a nonemphatic male study staff member they had not yet met that their performance in the math portion was not good enough and that they would need to repeat the task after the administration of the remaining tasks and questionnaires. Later in the session, participants were informed that repeating the task was not necessary because their performance was "good enough" (i.e., relief). Throughout the session, participants provided six saliva samples, eight VAMS ratings, and three self-reported measures of anxiety and affect.

the Partners Institutional Review Board. Next, participants completed two tasks, one probabilistic reward task (PRT) and one reaction time (RT) task (these data will be reported separately) and a clinical interview to determine eligibility (SCID). After the interview, participants completed the modified MAST, which was followed again by administration of the two tasks (Fig. 1). After this first laboratory session, participants were asked to return to the laboratory within $\sim 1$ month to complete an MRI scanning session (mean days $=25, \mathrm{SD}=21$ ). The scanning session included an fMRI task (described in Treadway et al., 2017), as well as a high-resolution anatomical scan.

MAST. Stress was induced via a modified version of the MAST (Smeets et al., 2012), a laboratory acute stress procedure that was shown previously to yield robust endocrine and affective stress responses among healthy individuals (Smeets et al., 2012). The MAST consists of a 5 min preparation phase and a $10 \mathrm{~min}$ acute stress phase that combines the physical aspects of immersing one hand in ice-cold water from the cold pressor test with the unpredictability, uncontrollability, negative social feedback, and mental arithmetic elements of the Trier Social Stress Test (see Smeets et al., 2012 for additional details). Unlike the original task, immediately upon MAST completion, participants were told by a nonemphatic male study staff they had not yet met that their performance in the math portion was not good enough and that they would need to repeat the task after the administration of the remaining tasks and questionnaires. This manipulation was intended to prolong the effect of the acute stressor. Later in the session, participants were informed that repeating the task was not necessary because their performance was "good enough" (i.e., relief was provided).

Stress measurements. To validate the effectiveness of the stress manipulation, endocrine and subjective indices of the stress response were assessed at multiple time points throughout the session. Saliva samples were taken at six time points: upon arrival $\left(T_{-80 \mathrm{~min}}\right)$, before the MAST $\left(T_{0 \text { min }}\right)$, slightly after its completion $\left(T_{+25 \mathrm{~min}}\right)$, and $50\left(T_{+50 \mathrm{~min}}\right), 65$ $\left(T_{+65 \mathrm{~min}}\right)$, and $100\left(T_{+100 \mathrm{~min}}\right)$ minutes after stress onset. These samples were used to track fluctuations in cortisol levels and $\alpha$-amylase, an established measure of the sympatho-adrenal-medullary adrenergic system (Nater and Rohleder, 2009). Saliva samples were obtained by placing a cotton swab in participants' mouths using Salivette collection devices (Sarstedt) and were stored at $-20^{\circ} \mathrm{C}$ until analysis. Cortisol and $\alpha$-amylase concentrations from saliva samples were assayed at the Laboratory for Biological Health Psychology at Brandeis University (Directors: Dr. Nicolas Rohleder and Dr. Jutta Wolf).

Affective responses to stress were assessed via a modified 100-point visual analog mood scale (VAMS) at the same six time points as the saliva collections, as well as at two additional times: before the clinical interview $\left(T_{-40 \mathrm{~min}}\right)$ and immediately after the relief $\left(T_{+90 \mathrm{~min}}\right)$. The VAMS consisted of five $100 \mathrm{~mm}$ horizontal lines, each representing a bipolar dimensional mood state: happy-sad, relaxed-tense, friendly-hostile, sociable-withdrawn, and quick witted-mentally slow. Participants indicated their response by moving a computer cursor on each line to the point that best described their current mood state. Such continuous, online assessment of mood was designed to avoid potential inaccuracies in retrospective poststress affect reporting (Hellhammer and Schubert, 2012). Finally, self-report measures of positive and negative affect (PANAS; Watson et al., 1988) and state anxiety (STAI-S; Spielberger et al., 1983) were assessed at three time points: upon arrival $\left(T_{-80 \mathrm{~min}}\right)$, after the MAST $\left(T_{+25 \mathrm{~min}}\right)$, and $100 \mathrm{~min}$ after stress onset $\left(T_{+100 \mathrm{~min}}\right)$ (Fig. 1).

MRI Acquisition and preprocessing. MRI data were acquired using a $3 \mathrm{~T}$ Siemens Tim Trio scanner and a 32-channel head coil at the McLean Imaging Center. Scanning protocol included high-resolution T1-weighted MPRAGE images $(\mathrm{TR}=2200 \mathrm{~ms} ; \mathrm{TE}=1.54 \mathrm{~ms} ; \mathrm{FOV}=230 \mathrm{~mm}$; matrix $=192 \times 192$; resolution $=1.22 \mathrm{~mm}^{3} ; 144$ slices $)$. MRI data were analyzed using the voxel-based morphometry (VBM) module of the Computational Anatomy Toolbox (CAT12) (http://www.neuro.unijena.de/cat/) for SPM12 (Wellcome Department of Cognitive Neurology). VBM analysis incorporated the following preprocessing steps: (1) spatial registration to a reference brain, (2) tissue classification (segmentation) into gray and white matter and CSF, (3) bias correction of intensity nonuniformities, and (4) smoothing $(8 \mathrm{~mm})$.

Statistical analysis. Of the 88 participants who were recruited, saliva samples of nine participants were excluded from analyses due to missing data, leaving a total sample size of 79 participants for cortisol and $\alpha$-amylase analyses. Ten additional participants did not undergo structural MRI, leaving a total sample size of 69 for MRI analyses. Cortisol responses were log transformed before statistical analysis to reduce skewness. Main effects of time were tested using a repeated-measures ANOVA with the six sampling time points as a within-subject factor. A similar approach was implemented for $\alpha$-amylase. Regarding the VAMS, ratings were transformed so that higher scores indicate greater negative affect. Further, to probe parallel endocrine and affective patterns, only the six VAMS ratings that were assessed alongside saliva samples were included in the analyses. VAMS ratings were analyzed using a repeated-measures ANOVA with the six sampling time points and the five VAMS scales as within-subject factors. Repeated-measures ANOVAs were also implemented separately for self-report measures of state anxiety (STAI-S), positive affect (PANAS-PA), and negative affect (PANAS-NA) with the three time points as a within-subject factor. For all post hoc tests, $\alpha$ was set at 0.05 and adjusted (Bonferroni) for multiple comparisons.

LCMM, also called the growth mixture model (Bauer and Curran, 2003), was used to identify distinct classes (i.e., groups) of participants featuring similar trajectories of cortisol response to stress. Specifically, we tested whether the model that best fits our data included two, three, or four classes of distinct trajectories of cortisol response. Within LCMM, time was modeled as polynomials while allowing for linear, quadratic, and cubic trajectories to be derived empirically based on trajectory subgroups. For continuous outcomes such as cortisol values, the LCMM is an extension of the standard linear mixed model for handling various subpopulations of longitudinal trajectories (O'Brien and Fitzmaurice, 2005). Importantly, this approach captures all the heterogeneity in individual trajectories and identifies subgroups of participants with similar profiles of trajectories independently of observed participant's characteristics. In other words, LCMM estimates the number of distinct trajectories of cortisol response that best capture variability in the data without requiring a priori assumptions regarding the number, size, or pattern of change of these trajectories. Bayesian information criterion (BIC) was used to compare different models, allowing for two versus three versus four classes and determine the optimal number based on variability in the data (Nylund et al., 2007). Low BIC values indicate a better fit of the model to the data. Analyses were performed using RStudio version 

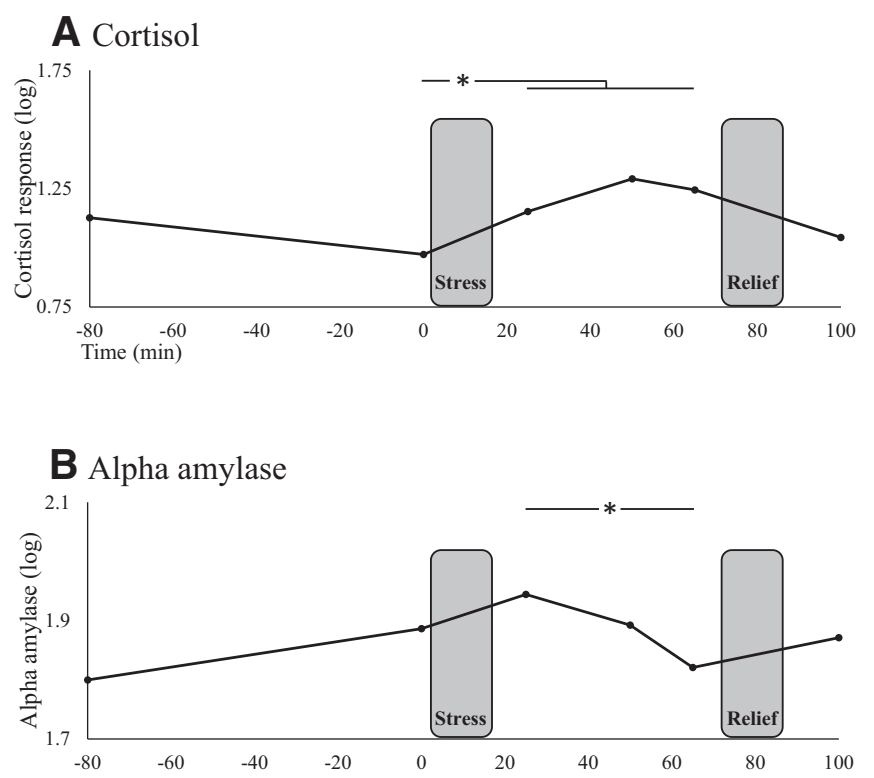
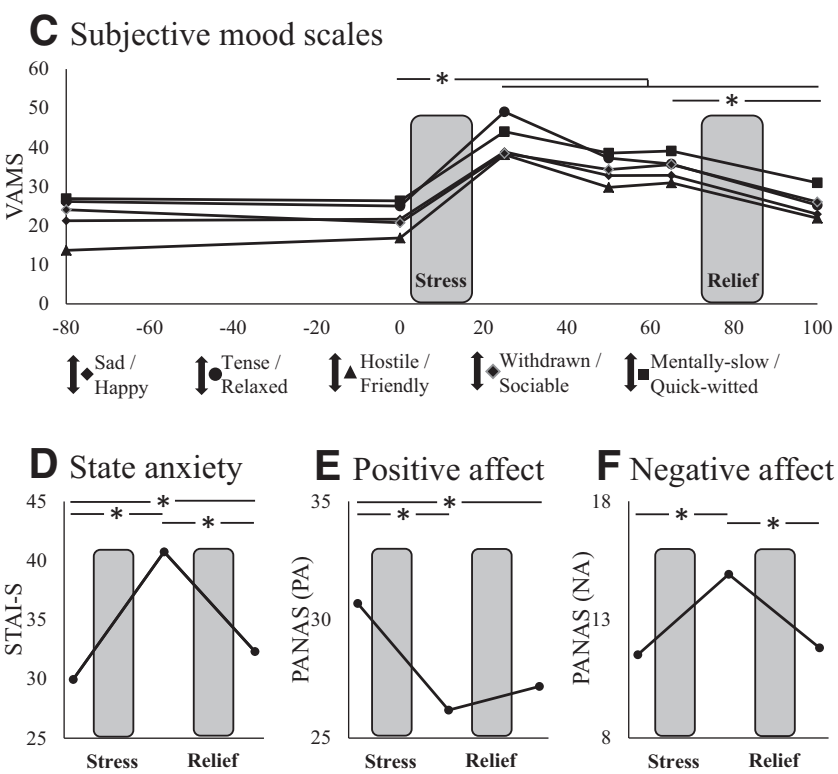

Figure 2. Overall effect of stress. Change in cortisol $(\boldsymbol{A}), \alpha$-amylase $(\boldsymbol{B})$, VAMS rating $(\boldsymbol{C})$, state anxiety ( $\boldsymbol{D})$, positive affect $(\boldsymbol{E})$, and negative affect $(\boldsymbol{F})$ throughout the session. Across all measures, there was a significant effect of time driven by stress induced increase in cortisol release and negative affect. Notably, cortisol levels and negative affect were still elevated 65 and 100 min after stress onset, respectively, reflecting a relatively prolonged acute stress induction. Participants were fully debriefed and their mood had returned to baseline before they left the laboratory. ${ }^{*} p<0.05$.

0.99 .879 (R Foundation) and the $\mathrm{lcmm}$ package (Proust-Lima et al., 2015). Notably, the first saliva sample, taken $\sim 80$ min before stress onset, was excluded from these analyses, leaving a total of five samples per participant. This was done in order to include in the models only stressrelated cortisol responses while avoiding endogenous variation in cortisol levels.

Next, cortisol and affective responses to stress were examined with regard to the three classes identified using LCMM. Notably, despite the fact that the LCMM models were defined based on cortisol data, running an ANOVA on cortisol patterns with the resulted classes as a factor is not circular and is in fact regarded as the most valid post hoc test (Jung and Wickrama, 2008). Accordingly, cortisol response to stress was examined using a mixed-effect ANOVA with the three classes as a between-subjects factor and the five sampling time points as a within-subjects factor (after omitting the first saliva sample, see above). For VAMS ratings, to compare only endocrine and affective data that were acquired concurrently, the first VAMS rating was used as an individual baseline score to which all other five VAMS samples were normalized. These data were then analyzed using a mixed-effect ANOVA with the three groups as a betweensubjects factor and the five sampling time points and five VAMS scales as within-subjects factors. Significant interactions were pursued for each VAMS scale separately using a mixed-effect ANOVA with groups $(n=3)$ and sampling time points $(n=5)$ as between- and within-subjects factors, respectively. Similarly, STAI-S, PANAS-PA, and PANAS-NA scores were examined separately using a mixed-effect ANOVA with groups $(n=3)$ and sampling time point $(n=3)$ as between- and within-subjects factors, respectively. For all post hoc tests, $\alpha$ was set at 0.05 and adjusted (Bonferroni) for multiple comparisons.

In addition to LCMM, cortisol response to stress was examined by calculating the area under the curve (AUC), a continuous measure that captures the total hormone concentration versus time-dependent change (Pruessner et al., 2003). Both AUC with respect to ground (AUCg) and AUC with respect to increase (AUCi) were computed because it was shown that these formulas may reveal different associations across variables (Pruessner et al., 2003). Finally, for descriptive purposes, a responder rate of participants showing a cortisol increase $\geq 2.5 \mathrm{nmol} / \mathrm{L}$ was also calculated.

For structural MRI, gray matter volumes were extracted for each participant from anatomical masks of the amygdala and hippocampus following a priori hypotheses relating to their key role in HPA regulation. Potential relations between amygdala and hippocampus gray matter vol- umes and cortisol response to stress were examined using a mixed-effect ANOVA with the three cortisol classes as a between-subjects factor and left and right amygdala and hippocampus gray matter volumes as a within-subjects factor (separately per region). In addition, exploratory analyses were also conducted to compare gray matter volumes of the hippocampus and amygdala with continuous indices of cortisol release (individual AUCi and AUCg values) using hierarchical regression with linear regression in the first step and quadratic regression in the second step such that a significant $F$ change would indicate a quadratic effect. Given a total of eight regression analyses (2 regions, 2 sides, 2 AUC measures), the $p$ value for significance was set at $0.05 / 8=0.00625$ (Bonferroni corrected).

\section{Results}

\section{Overall effect of stress}

Repeated-measures ANOVA with cortisol responses to stress revealed a main effect of time $\left(F_{(5,390)}=47.25, p<0.001\right)$, with a strong quadratic effect $\left(F_{(1,78)}=40.70, p<0.001\right)$. Post hoc analyses revealed significant stress-induced increase in mean cortisol levels from before the MAST $\left(T_{0 \text { min }}\right)$ to $25\left(T_{+25 \mathrm{~min}}\right), 50\left(T_{+50 \mathrm{~min}}\right)$, and $65 \mathrm{~min}\left(T_{+65 \mathrm{~min}}\right)($ all $p<0.001)$, but not $100 \mathrm{~min}$ after its onset $\left(T_{+100 \mathrm{~min}} ; p=0.2\right)$ (Fig. $\left.2 A\right)$. For $\alpha$-amylase, the main effect of time was also significant $\left(F_{(5,390)}=9.29, p<0.001\right)$. Post hoc tests revealed some increase from before the MAST to slightly after its completion, although it did not reach the significance level $\left(T_{+25 \min } ; p=0.151\right)$. There was, however, a significant decrease in $\alpha$-amylase levels from $25 \mathrm{~min}$ after MAST onset to 40 min later $\left(T_{+25 \text { min }}>T_{+65 \text { min }} ; p<0.001\right.$; Fig. $\left.2 B\right)$.

When considering VAMS ratings, repeated-measures ANOVA also resulted in a highly significant main effect of time, indicating an overall increase in negative affect across all five VAMS scales $\left(F_{(5,400)}=96.93, p<0.001\right)$, with the expected quadratic effect $\left(F_{(1,80)}=124.40, p<0.001\right)$. Mirroring the cortisol results, negative mood across all scales was elevated $25\left(T_{+25 \mathrm{~min}}\right), 50\left(T_{+50 \mathrm{~min}}\right)$, and $65 \mathrm{~min}\left(T_{+65 \mathrm{~min}}\right)$ after stress onset relative to before stress $\left(T_{0 \mathrm{~min}}\right)$ (all $p<0.001)$. Furthermore, unlike cortisol, negative mood was still elevated at the final sampling time point $100 \mathrm{~min}$ after stress onset relative to before stress $\left(T_{+100 \min }>T_{0 \mathrm{~min}} ; p<0.001\right)$, yet it was 
significantly less negative at that point relative to $65 \mathrm{~min}$ after stress onset (after an additional $35 \mathrm{~min}$ that included the relief component; $T_{+65 \text { min }}>T_{+100 \min } ; p<$ 0.001) (Fig. 2C).

Repeated-measures ANOVA on selfreported anxiety state (STAI-S) revealed a main effect of time $\left(F_{(2,162)}=80.62, p<\right.$ $0.001)$ due to significantly higher anxiety levels after the MAST $\left(T_{+25} \mathrm{~min}\right)$ compared with both arrival $\left(T_{-80 \mathrm{~min}}\right)$ and session completions $\left(T_{+100 \mathrm{~min}}\right)$ (all $p<$ 0.001 ). Interestingly, anxiety levels were still significantly higher at session completion relative to arrival $\left(T_{+100 \mathrm{~min}}>T_{-80 \mathrm{~min}} ; p=\right.$ 0.004; Fig. 2D). A similar analysis for positive affect (PANAS-PA) also revealed a main effect of time $\left(F_{(2,162)}=29.52, p<0.001\right)$ that was driven by significant reduction in positive affect after the MAST compared with arrival $\left(T_{+25}\right.$ min $<T_{-80 \text { min }} ; p<$ $0.001)$, a reduction that was still evident at session completion $\left(T_{+100 \min }<T_{-80 \mathrm{~min}}\right.$; $p<0.001$ ) (Fig. 2E). Correspondingly, a main effect of time $\left(F_{(2,162)}=38.88, p<\right.$ $0.001)$ for negative affect (PANAS-NA) was found to be driven by significantly higher negative affect after the MAST compared with both arrival and session completion $\left(T_{+25 \text { min }}>T_{-80 \text { min }} ; T_{+25 \text { min }}\right.$ $>T_{+100 \mathrm{~min}}$; all $p<0.001$ ) (Fig. $2 F$ ). See Treadway et al. (2017) for additional analyses from this cohort.

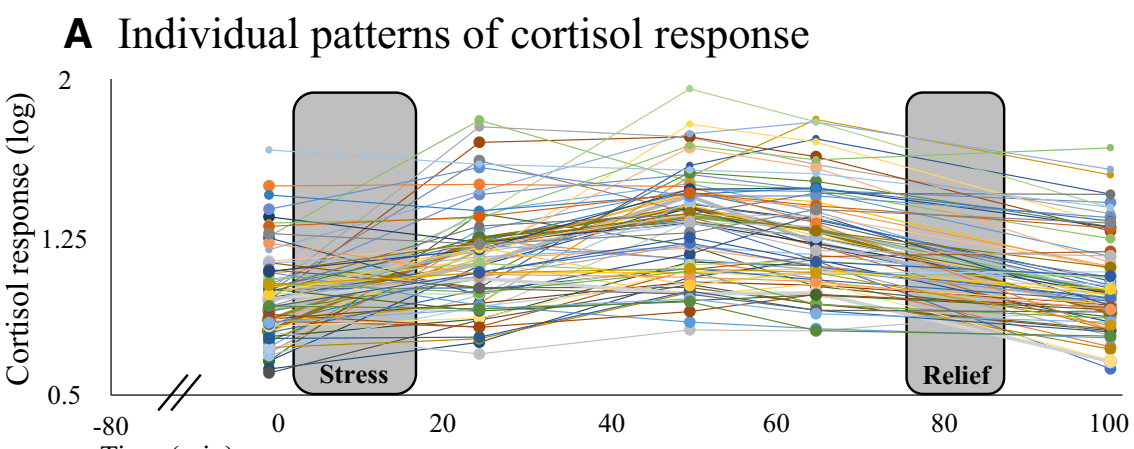

Time (min)

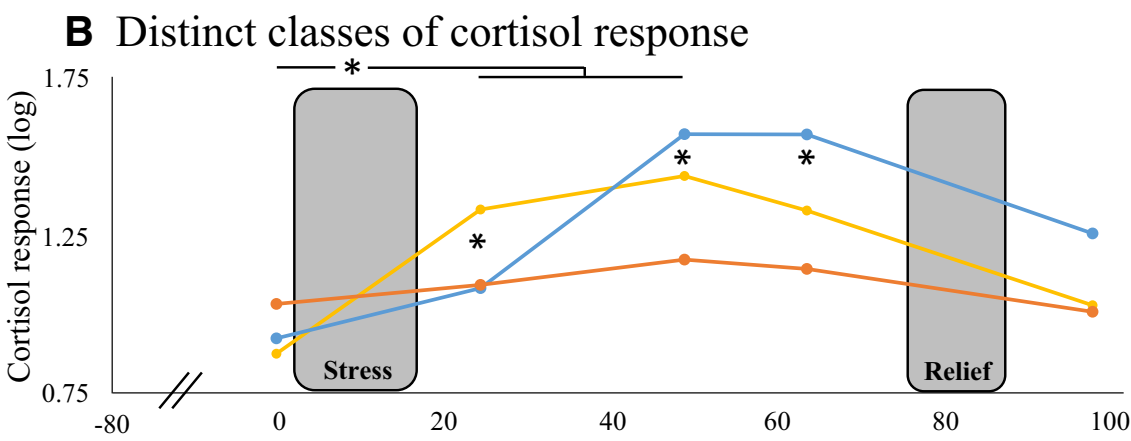

- Hyper-response $(n=10) \bullet$ Moderate-response $(n=21) \bullet$ Mild-response $(n=48)$

Figure 3. LCMM. $\boldsymbol{A}$, Individual patterns of cortisol response throughout the session $(n=79)$. $\boldsymbol{B}$, Applying LCMM on these data revealed that the model that best fit our data included three latent classes, labeled based on their distinct trajectories of cortisol response to stress as the hyper-response $(n=10)$, moderate-response $(n=21)$, and mild-response $(n=48)$ groups. Note that all three groups exhibited a significant stress-induced increase in cortisol release 25 and 50 min after stress onset. ${ }^{*} p<0.05$.

\section{LCMM}

Approximately $72 \%$ of participants from our sample (57/79) could be classified as cortisol responders based on a cortisol increase $\geq 2.5 \mathrm{nmol} / \mathrm{L}$, suggesting that the laboratory procedure succeeded in activating HPA response. Despite these robust overall effects, patterns of cortisol response throughout the session greatly differed across participants, showing various patterns of change over time (Fig. 3A). Accordingly, individual cortisol response patterns were analyzed via LCMM aiming to estimate whether two, three, or four distinct trajectories of cortisol response best captured variability in the data without requiring a priori assumptions. BIC criteria comparing the different LCMM models yielded $\mathrm{BIC}_{2}=424, \mathrm{BIC}_{3}=413$, and $\mathrm{BIC}_{4}=416$, indicating that a model allowing for three latent classes was optimal. These three classes were labeled based on their distinct trajectories of cortisol response to stress as the hyper-response $(n=10)$, moderate-response $(n=21)$, and mild-response $(n=48)$ groups. Figure $3 B$ depicts the estimated mean trajectories of the three groups. Note that in the second-best model (4-class model), the fourth class was small ( $7.6 \%$ of the sample), thus contributing very little value beyond the 3 -class model. The model allowing for three latent classes also had a good discrimination ability, with $<15 \%$ of participants a posteriori classified in other classes than the one initially assigned (see Table 2 for full statistic description).

Mixed-effect ANOVA with the three groups from LCMM as a between-subjects factor and the five cortisol sampling time points as a within-subjects factor revealed, as expected, a significant
Table 2. LCMM model statistics

\begin{tabular}{|c|c|c|c|c|c|c|c|}
\hline \multicolumn{8}{|c|}{ Between-model comparison } \\
\hline & $\begin{array}{l}\text { Log } \\
\text { likelihood }\end{array}$ & $\mathrm{NPM}^{a}$ & $\mathrm{BIC}$ & Class $1^{b}$ & Class $2^{b}$ & Class $3^{b}$ & Class $4^{b}$ \\
\hline 2 Classes & -185.89 & 12 & 424.22 & $40.5 \%$ & $59.5 \%$ & & \\
\hline 3 Classes & -169.81 & 17 & 413.89 & $26.6 \%$ & $12.7 \%$ & $60.7 \%$ & \\
\hline 4 Classes & -159.67 & 22 & 415.47 & $7.6 \%$ & $26.6 \%$ & $11.4 \%$ & $54.4 \%$ \\
\hline \multicolumn{8}{|c|}{$\begin{array}{l}\text { Within the three-class model: fixed effects allowing for cubic time }(t) \text { trends } \\
\end{array}$} \\
\hline & \multicolumn{2}{|l|}{$n(\%)$} & \multicolumn{2}{|l|}{ Coefficient } & SE & Wald & $p$ \\
\hline \multirow[t]{3}{*}{ Class 1} & \multirow[t]{3}{*}{$21(26)$} & $t$ & 8.47 & & & 2.78 & 0.0055 \\
\hline & & $t^{2}$ & 32.88 & & & 5.97 & $<0.0001$ \\
\hline & & $t^{3}$ & 0.13 & & & 0.07 & 0.9441 \\
\hline \multirow[t]{3}{*}{ Class 2} & \multirow[t]{3}{*}{$10(13)$} & $t$ & -40.52 & & & -11.69 & $<0.0001$ \\
\hline & & $t^{2}$ & -34.27 & & & -8.67 & $<0.0001$ \\
\hline & & $t^{3}$ & -12.14 & & & -7.51 & $<0.0001$ \\
\hline \multirow[t]{3}{*}{ Class 3} & \multirow[t]{3}{*}{$48(61)$} & $t$ & 4.29 & & & 1.71 & 0.0878 \\
\hline & & $t^{2}$ & -17.07 & & & -4.25 & 0.0002 \\
\hline & & $t^{3}$ & -3.97 & & & -2.92 & 0.0035 \\
\hline \multicolumn{8}{|c|}{ Within the three-class model: mean of posterior probabilities (\%) in each class } \\
\hline & & \multicolumn{2}{|r|}{ Class 1} & \multicolumn{3}{|c|}{ Class 2} & Class 3 \\
\hline \multicolumn{2}{|c|}{ Class $1(n=21)$} & \multicolumn{2}{|c|}{91} & \multicolumn{3}{|c|}{4} & 5 \\
\hline \multirow{2}{*}{\multicolumn{2}{|c|}{$\begin{array}{l}\text { Class } 2(n=10) \\
\text { Class } 3(n=48)\end{array}$}} & \multicolumn{2}{|r|}{$\begin{array}{l}8 \\
4\end{array}$} & \multicolumn{3}{|c|}{$\begin{array}{r}85 \\
1\end{array}$} & 7 \\
\hline & & & 4 & & 1 & & 95 \\
\hline
\end{tabular}

${ }^{a}$ Number of model parameters.

${ }^{b}$ Posterior proportion for each class. 
A Sad / Happy by cortisol class

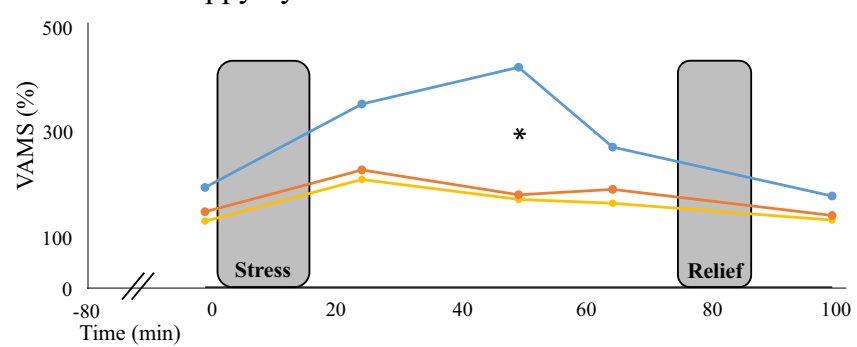

B Tense / Relaxed by cortisol class

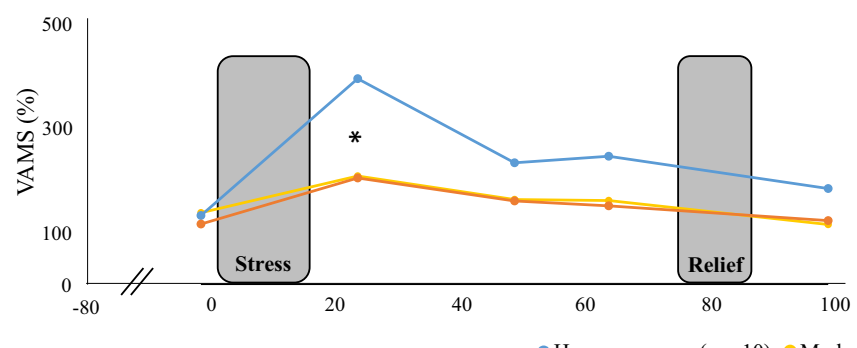

C Hostile / Friendly by cortisol class
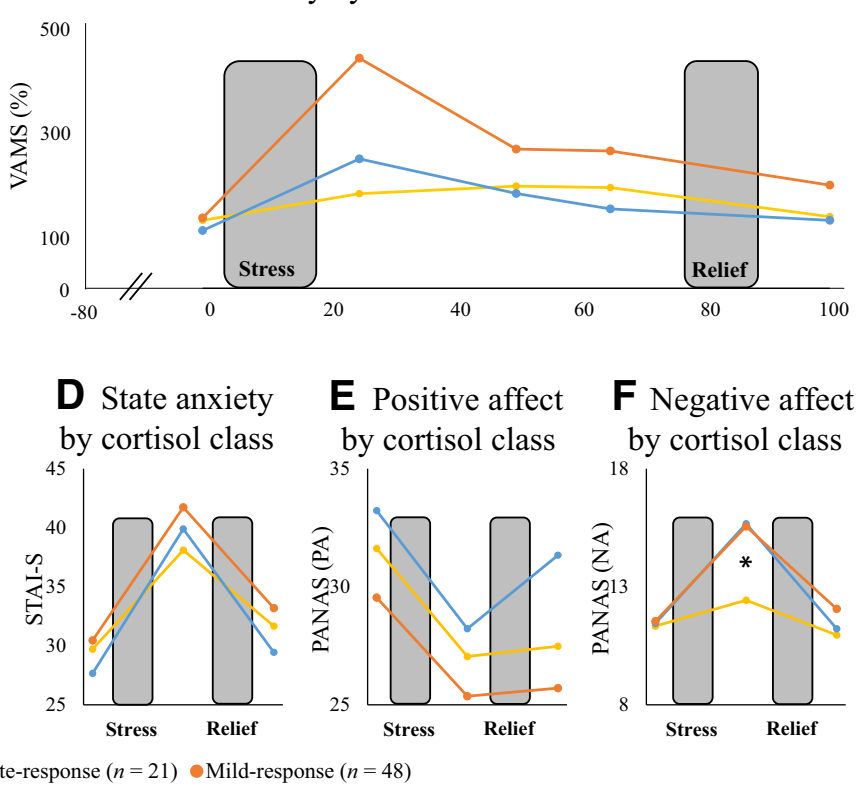

$\mathbf{F}$ Negative affect by cortisol class

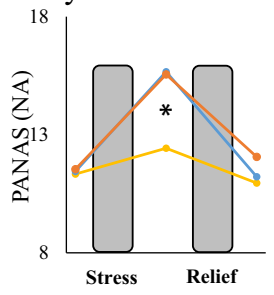

- Hyper-response $(n=10) \bullet$ Moderate-response $(n=21) \bullet$ Mild-response $(n=48)$

Figure 4. Effects of stress by cortisol class. Change in happy-sad scale $(\boldsymbol{A})$, relaxed-tense scale $(\boldsymbol{B})$, friendly-hostile scale $(\boldsymbol{C})$, state anxiety $(\boldsymbol{D})$, positive affect $(\boldsymbol{E})$, and negative affect $(\boldsymbol{F})$ throughout the session as a function of cortisol class. Note that individuals in the moderate response class exhibited less stress-induced sadness and tension relative to individuals in the hyper-response class, as well as less hostility (trend) and negative affect relative to individuals in the mild-response class. ${ }^{*} p<0.05$.

main effect of time, which was not pursued by post hoc tests. The ANOVA further revealed a main effect of groups $\left(F_{(2,76)}=6.17, p=\right.$ 0.003 ) due to significantly higher overall cortisol release in the hyperresponse class relative to the mild-response class $(p=0.009)$. Critically, a significant group $\times$ time interaction $\left(F_{(8,304)}=36.92, p<\right.$ 0.001 ) also emerged, which was followed up by within- and betweengroup post hoc comparisons. Within-group analyses raveled a significant stress-induced increase in mean cortisol release from before the MAST $\left(T_{0 \mathrm{~min}}\right)$ to both $25\left(T_{+25 \mathrm{~min}}\right)$ and $50\left(T_{+50 \mathrm{~min}}\right)$ minutes after MAST onset (all $p<0.05$ ) in all three classes (Fig. $3 B$ ). Between-groupcomparison, however, revealed that themoderate-response class exhibited significantly increased cortisol release 25 min after MAST onset $\left(T_{+25}\right.$ min $)$ relative to both the mildresponse and hyper-response classes $(p<0.001$ and $p=0.008$, respectively), whereas the hyper-response class exhibited significantly increased cortisol release $50\left(T_{+50 \mathrm{~min}}\right)$ and $65\left(T_{+65 \mathrm{~min}}\right)$ minutes after MAST onset relative to both the mild-response and moderate-response classes (all $p<0.001$ ) (Fig. 3B).

\section{Effects of stress by cortisol class}

When considering the VAMS ratings of the three LCMM groups, mixed-effect ANOVA revealed a main effect of time $\left(F_{(4,304)}=\right.$ $23.96, p<0.001)$, a group $\times$ scale interaction $\left(F_{(8,1216)}=2.74\right.$, $p=0.006)$, and a group $\times$ time $\times$ scale interaction $\left(F_{(32,1216)}=\right.$ $2.46, p<0.001$ ), which was pursued for each scale separately. This resulted in three of the five scales showing a significant group $\times$ time interaction: $\left(\right.$ happy-sad, $F_{(8,304)}=2.06, p=0.039$; relaxed-tense, $F_{(8,304)}=3.10, p=0.002$; friendly-hostile, $F_{(8,304)}=$ $2.15, p=0.031)$. For the happy-sad scale, post hoc tests indicated that these results were driven by significantly higher sadness scores $50 \mathrm{~min}\left(T_{+50 \mathrm{~min}}\right)$ after stress onset in the hyper-response class relative to both the mild-response and moderate-response classes $(p=0.006, p=0.003$, respectively) (Fig. $4 A)$. A similar pattern emerged with regard to the relaxed-tense scale except that increased tension among hyper-response relative to the mildresponse and moderate-response classes occurred $25 \mathrm{~min}$ after stress onset $\left(T_{+25 \mathrm{~min}}\right)(p=0.05$ and $p=0.023$, respectively; Fig. $4 B)$. For the friendly-hostile scale, the analysis revealed that the mild-response class was driving the effect, exhibiting a trend toward more hostility $25 \mathrm{~min}$ after stress onset $\left(T_{+25 \mathrm{~min}}\right)$ compared with the moderate-response class ( $p=0.080$; Fig. $4 C)$. For state anxiety (STAI-S) and positive affect (PANAS-PA), the mixedeffect ANOVAs with LCMM groups revealed no effect of groups (Fig. $4 D, E$ ). For negative affect (PANAS-NA), however, there was a significant group $\times$ time interaction $\left(F_{(4,148)}=3.17, p=\right.$ 0.016 ) driven by significantly more negative affect $25 \mathrm{~min}$ after stress onset in the mild-response class compared with the moderate-response class $(p=0.037)$ (Fig. $4 F)$.

\section{Structural variability and cortisol class}

Mixed-effect ANOVA with the three groups from LCMM as a between-subjects factor and left and right hippocampal gray matter volume as a within-subjects factor revealed only a significant main effect of side due to overall more gray matter volume on the right compared with the left side $\left(F_{(1,66)}=33.52, p<0.001\right)$, with no group or group $\times$ side effects $(p=0.99$ and $p=0.36$, respectively, data not shown). Analyses of amygdala gray matter volume yielded similar results, including a main effect of side (right $>$ left; $\left.F_{(1,66)}=138.04, p<0.001\right)$ and no group or group $\times$ side effects ( $p=0.99, p=0.16$, respectively, data not shown).

\section{Structural variability and cortisol AUC}

Hierarchical regressions with the continuous cortisol response measure AUCg separately for participants' gray matter volumes in the left and right amygdala and hippocampus revealed a significant relation only for the right hippocampus. Further, a significant $F$ change in the hierarchical regression model $\left(F_{(1,66)}=\right.$ 12.56, $p=0.001$; significant after Bonferroni correction; $p<$ 0.00625 ) indicated that high and low AUCg values were both associated with less right hippocampal gray matter volume compared with moderate AUCg levels $(r=0.421$; Fig. 5). Similar results appeared when using AUCi as the dependent measure in 
the regression, showing only a significant, quadratic relation between AUCi magnitude and right hippocampal gray matter volume $\left(F_{(1,66)}=4.86, p=0.031 ; r=\right.$ 0.262). AUCi results, however, did not survive multiple-comparisons correction and should therefore be considered with caution.

\section{Discussion}

The overarching goal of the current study was threefold. First, we aimed to evaluate an acute stress laboratory procedure specifically designed to yield a prolonged effect. Toward this end, participants completed the MAST, a robust laboratory procedure for acute stress induction (Smeets et al., 2012), and were told immediately afterward that they would soon need to repeat the task due to their poor performance. Results indicated that this revised version of the MAST yielded stressinduced increase in cortisol release 25,50, and $65 \mathrm{~min}$ after stress onset, as well as a shift toward more negative affect in all of these time points and up to $100 \mathrm{~min}$ after stress onset. This represents a relatively prolonged period of acute stress induction compared with previous reports, including studies using the original MAST procedure. Given the inherent delay between stress onset and increase in salivary cortisol and uncertainties whether individuals differ in such latency, efficient manipulations that induce a sustained acute stress response could be useful in future research.

\section{Trajectories of cortisol response to prolonged acute stress}

By probing individual patterns of stress-induced cortisol release over this relatively prolonged time period and analyzing these patterns using LCMM, our second aim was to investigate the number of distinct trajectories of cortisol response that best captured variability in our data without a priori assumptions. This analysis revealed three distinct trajectories of cortisol response, labeled as the hyper-response, moderateresponse, and mild-response groups, all exhibiting a significant, quadratic increase in cortisol release. Generalization of this finding should proceed with caution. First, because only healthy females were included, no predictions can be made regarding cortisol patterns that may emerge in response to stress in males or among psychiatric samples. More critically, it is possible that specific sample characteristics influenced the final number of classes identified. In fact, we expect that a higher number of cortisol trajectories may emerge among larger and more environmentally and/or genetically diverse samples. Although the exact number of classes may vary based on sample characteristics, our results point to the importance of adequately modeling individual differences in cortisol response to stress and including multiple measures of the response trajectory when probing the endocrine stress response. The endocrine stress-response system, most prominently the HPA axis, acts via tightly regulated negative feedback loops that control the onset, magnitude, and duration of stress response activation (Joëls and Baram, 2009). Accordingly, these different parameters of the stress response system may all contribute to determine its physiological and affective outcomes.

\section{Linking cortisol and affective responses to prolonged acute stress}

In parallel with variability in cortisol stress response patterns, individuals are also known to differ in their affective responses to stress, including in measures of response threshold, amplitude, and rise time to peak (Davidson, 2000). In the current study, we found that, although all three cortisol groups experienced a shift toward a more negative affective state, individuals in the moderateresponse class exhibited less stress-induced sadness and tension relative to individuals in the hyper-response class, as well as less hostility (trend) and negative affect relative to individuals in the mild-response class. Such quadratic association between the magnitude of cortisol response to stress and affective responses may account for the paucity of studies reporting a linear link between the two (Campbell and Ehlert, 2012). This quadratic pattern is also consistent with the well established inverted U-shape relationship between basal levels of cortisol and cognitive performance, with beneficial effects of moderately elevated cortisol levels on cognition (de Kloet et al., 1999). Why both "too little" and "too much" cortisol are associated with reduced cognitive performance and/or heightened affective response to stress is not clear. One potential explanation may relate to the notion that, at least for some susceptible individuals, repeated hypersecretion of cortisol may have caused desensitization of the HPA axis, eventually resulting in reduced HPA sensitivity (Heim et al., 2000). This interpretation remains speculative in regard to our sample, however, because we did not assess participants' lifetime exposure to stress. It has been suggested that lifetime exposure to stress itself may affect stress vulnerability via a quadratic pattern, challenging the assumption that stress and negative outcomes show a simple, linear relationship (Liu, 2015). Our findings suggest that cortisol and affective response to stress may also exhibit a nonlinear relation.

\section{Linking cortisol responses to prolonged acute stress and hippocampal structural variability}

The third aim of the current study was to investigate potential relations between cortisol response to stress and structural variability in the amygdala and hippocampus, key HPA regulatory regions. Resembling the link between cortisol and affective responses, a quadratic link was also present between a continuous measure of cortisol response to stress (AUCg) and hippocampal gray matter volume. Specifically, both high and low levels of stress-induced cortisol release were found to be associated with 
less right hippocampal gray matter volume compared with moderate cortisol release. This finding may be discussed in light of extensive preclinical evidence showing that exposure to cumulative stress may translate to morphological damage, most pronouncedly in the hippocampus (Sapolsky et al., 1990; Joëls et al., 2004). Increased susceptibility of the hippocampus to the effects of stress was attributed to its important role in stress regulation, as reflected by its substantial number of cortisol receptors (McEwen, 1999). In humans, reduced hippocampal volume has been the most commonly described neural structural abnormality in people who were exposed to traumatic stress and consequently developed an anxiety disorder such as posttraumatic stress disorder (Smith, 2005). Accordingly, reduced hippocampal volume among individuals exhibiting mild cortisol response may further link such a response pattern to repeated hypersecretion of cortisol. This interpretation, again, could be tested in studies assessing participants' lifetime exposure to stress.

\section{Limitations and conclusions}

A few limitations of our study should be emphasized. First, it is not clear why $\alpha$-amylase levels did not increase in response to stress in the current study nor why only some affective scales showed a significant relation with cortisol trajectories whereas others did not. Second, our LCMM-based classes did not differ in their hippocampal and amygdala gray matter volumes and the link between cortisol response and hippocampal volume only emerged when treating cortisol response as a continuous measure. This could relate to our modest sample size for these analyses, with only seven participants with MRI data in the hyper-response class (20 in the moderate-response class and 42 in the mild-response class). Whether the link between cortisol response to prolonged acute stress, affective responses, and hippocampal gray matter volume is categorical or continuous by nature is a topic for future research. A third limitation relates to the fact that factors that were shown previously to affect emotional and cortisol responses to acute psychosocial stressor in healthy volunteers, including menstrual cycle phase (Duchesne and Pruessner, 2013), contraceptive usage (Roche et al., 2013), and personality traits (Childs et al., 2014), did not interact with cortisol classes in the current study ( $\chi^{2}$ tests revealed no effects of menstrual cycle phase or contraceptives use on cortisol class: $\chi^{2}=1.82$ and 2.83, respectively; mixed-effect ANOVA revealed no interaction of NEO fivefactor personality scores with cortisol class: $p=0.82$ ). These inconsistencies could stem from the fact that previous work did not apply LCMM to classify cortisol response, but rather relied on mean group measures. Alternately, it may also be the case that additional factors we did not control for may have influenced current results, including age (Hostinar et al., 2014), fatigue (Bower et al., 2005) and body shape (Epel et al., 2000), to name a few. Whereas controlling for all potential contributors to the stress response is practically impossible, future studies may choose to assess multiple factors and try to incorporate them into multidimensional models to account for additional variability in the data.

Our results suggest that investigating cortisol responses to stress without a priori assumptions regarding pattern of change can uncover distinct trajectories of cortisol response to prolonged acute stress among healthy females that would have been overlooked in conventional analyses parsing participants into cortisol responders and nonresponders. Most critically, identifying such distinct cortisol trajectories enabled us to discover the oftenhidden link between stress-induced cortisol release and patterns of affective responses to stress as well as hippocampal structural variability. Given that neuroendocrine stress responses are conceptualized as biomarkers reflecting individual differences in stress resilience and susceptibility to psychopathology and disease (Feder et al., 2009), these insights regarding individual differences in trajectories of cortisol response to stress may have clinical implications.

\section{References}

Bauer DJ, Curran PJ (2003) Distributional assumptions of growth mixture models: implications for overextraction of latent trajectory classes. Psychol Methods 8:338-363. CrossRef Medline

Blascovich JJ, Vanman E, Mendes WB, Dickerson S (2011) Social psychophysiology for social and personality psychology. Thousand Oaks, CA: Sage.

Bower JE, Ganz PA, Aziz N (2005) Altered cortisol response to psychologic stress in breast cancer survivors with persistent fatigue. Psychosom Med 67:277-280. CrossRef Medline

Cacciaglia R, Nees F, Grimm O, Ridder S, Pohlack ST, Diener SJ, Liebscher C, Flor H (2017) Trauma exposure relates to heightened stress, altered amygdala morphology and deficient extinction learning: implications for psychopathology. Psychoneuroendocrinology 76:19-28. CrossRef Medline

Campbell J, Ehlert U (2012) Acute psychosocial stress: does the emotional stress response correspond with physiological responses? Psychoneuroendocrinology 37:1111-1134. CrossRef Medline

Childs E, White TL, de Wit H (2014) Personality traits modulate emotional and physiological responses to stress. Behav Pharmacol 25:493-502. CrossRef Medline

Cho K (2001) Chronic 'jet lag' produces temporal lobe atrophy and spatial cognitive deficits. Nat Neurosci 4:567-568. CrossRef Medline

Davidson RJ (2000) Affective style, psychopathology, and resilience: brain mechanisms and plasticity. Am Psychol 55:1196-1214. CrossRef Medline

de Kloet ER, Oitzl MS, Joëls M (1999) Stress and cognition: are corticosteroids good or bad guys? Trends Neurosci 22:422-426. CrossRef Medline

Dickerson SS, Kemeny ME (2004) Acute stressors and cortisol responses: a theoretical integration and synthesis of laboratory research. Psychol Bull 130:355-391. CrossRef Medline

Duchesne A, Pruessner JC (2013) Association between subjective and cortisol stress response depends on the menstrual cycle phase. Psychoneuroendocrinology 38:3155-3159. CrossRef Medline

Epel ES, McEwen B, Seeman T, Matthews K, Castellazzo G, Brownell KD, Bell J, Ickovics JR (2000) Stress and body shape: stress-induced cortisol secretion is consistently greater among women with central fat. Psychosom Med 62:623-632. CrossRef Medline

Feder A, Nestler EJ, Charney DS (2009) Psychobiology and molecular genetics of resilience. Nat Rev Neurosci 10:446-457. CrossRef Medline

First MB, Spitzer RL, Gibbon M, Williams JBW (2005) Structured clinical interview for DSM-IV-TR axis I disorders: patient edition (with psychotic screen). New York, Biometrics Research, New York State Psychiatric Institute; 2002.

Foley P, Kirschbaum C (2010) Human hypothalamus-pituitary-adrenal axis responses to acute psychosocial stress in laboratory settings. Neurosci Biobehav Rev 35:91-96. CrossRef Medline

Harkness KL, Hayden EP, Lopez-Duran NL (2015) Stress sensitivity and stress sensitization in psychopathology: an introduction to the special section. J Abnorm Psychol 124:1-3. CrossRef Medline

Heim C, Ehlert U, Hellhammer DH (2000) The potential role of hypocortisolism in the pathophysiology of stress-related bodily disorders. Psychoneuroendocrinology 25:1-35. CrossRef Medline

Hellhammer J, Schubert M (2012) The physiological response to Trier Social Stress Test relates to subjective measures of stress during but not before or after the test. Psychoneuroendocrinology 37:119-124. CrossRef Medline

Herman JP, McKlveen JM, Ghosal S, Kopp B, Wulsin A, Makinson R, Scheimann J, Myers B (2016) Regulation of the hypothalamic-pituitaryadrenocortical stress response. Compr Physiol 6:603-621. CrossRef Medline

Hostinar CE, McQuillan MT, Mirous HJ, Grant KE, Adam EK (2014) Cortisol responses to a group public speaking task for adolescents: variations by age, gender, and race. Psychoneuroendocrinology 50: 155-166. CrossRef Medline 
Joëls M, Baram TZ (2009) The neuro-symphony of stress. Nat Rev Neurosci 10:459-466. CrossRef Medline

Joëls M, Karst H, Alfarez D, Heine VM, Qin Y, van Riel E, Verkuyl M, Lucassen PJ, Krugers HJ (2004) Effects of chronic stress on structure and cell function in rat hippocampus and hypothalamus. Stress 7:221-231. CrossRef Medline

Jung T, Wickrama KAS (2008) An introduction to latent class growth analysis and growth mixture modeling. Social and Personality Psychology Compass 2:302-317. CrossRef Medline

Klimes-Dougan B, Eberly LE, Westlund Schreiner M, Kurkiewicz P, Houri A, Schlesinger A, Thomas KM, Mueller BA, Lim KO, Cullen KR (2014) Multilevel assessment of the neurobiological threat system in depressed adolescents: interplay between the limbic system and hypothalamicpituitary-adrenal axis. Dev Psychopathol 26:1321-1335. CrossRef Medline

Kudielka BM, Kirschbaum C (2005) Sex differences in HPA axis responses to stress: a review. Biol Psychol 69:113-132. CrossRef Medline

Liu J, Chaplin TM, Wang F, Sinha R, Mayes LC, Blumberg HP (2012) Stress reactivity and corticolimbic response to emotional faces in adolescents. J Am Acad Child Adolesc Psychiatry 51:304-312. CrossRef Medline

Liu RT (2015) A developmentally informed perspective on the relation between stress and psychopathology: when the problem with stress is that there is not enough. J Abnorm Psychol 124:80-92. CrossRef Medline

McEwen BS (1999) Stress and hippocampal plasticity. Annu Rev Neurosci 22:105-122. CrossRef Medline

Monroe SM, Simons AD (1991) Diathesis-stress theories in the context of life stress research: implications for the depressive disorders. Psychol Bull 110:406-425. CrossRef Medline

Nater UM, Rohleder N (2009) Salivary alpha-amylase as a non-invasive biomarker for the sympathetic nervous system: current state of research. Psychoneuroendocrinology 34:486-496. CrossRef Medline

Nylund KL, Asparouhov T, Muthén BO (2007) Deciding on the number of classes in latent class analysis and growth mixture modeling: a Monte Carlo simulation study. Structural Equation Modeling: A Multidisciplinary Journal 14:535-569. CrossRef

O'Brien LM, Fitzmaurice GM (2005) Regression models for the analysis of longitudinal Gaussian data from multiple sources. Stat Med 24:17251744. CrossRef Medline
Proust-Lima C, Philipps V, Liquet B (2015) Estimation of extended mixed models using latent classes and latent processes: the $\mathrm{R}$ package $\mathrm{lcmm}$. arXiv 1503.00890.

Pruessner JC, Kirschbaum C, Meinlschmid G, Hellhammer DH (2003) Two formulas for computation of the area under the curve represent measures of total hormone concentration versus time-dependent change. Psychoneuroendocrinology 28:916-931. CrossRef Medline

Pruessner M, Pruessner JC, Hellhammer DH, Bruce Pike G, Lupien SJ (2007) The associations among hippocampal volume, cortisol reactivity, and memory performance in healthy young men. Psychiatry Res 155:1-10. CrossRef Medline

Roche DJ, King AC, Cohoon AJ, Lovallo WR (2013) Hormonal contraceptive use diminishes salivary cortisol response to psychosocial stress and naltrexone in healthy women. Pharmacol Biochem Behav 109:84-90. CrossRef Medline

Sapolsky RM, Uno H, Rebert CS, Finch CE (1990) Hippocampal damage associated with prolonged glucocorticoid exposure in primates. J Neurosci 10:2897-2902. Medline

Smeets T, Cornelisse S, Quaedflieg CW, Meyer T, Jelicic M, Merckelbach H (2012) Introducing the Maastricht Acute Stress Test (MAST): A quick and non-invasive approach to elicit robust autonomic and glucocorticoid stress responses. Psychoneuroendocrinology 37:1998-2008. CrossRef Medline

Smith ME (2005) Bilateral hippocampal volume reduction in adults with post-traumatic stress disorder: a meta-analysis of structural MRI studies. Hippocampus 15:798-807. CrossRef Medline

Spielberger CD, Gorsuch RL, Lushene R, Vagg IR, Jacobs GA (1983) Manual for the state-trait anxiety inventory. Palo Alto, CA: Consulting Psychologist.

Treadway MT, Admon R, Arulpragasam AR, Mehta M, Douglas S, Vitaliano G, Olson DP, Cooper JA, Pizzagalli DA (2017) Association between interleukin- 6 and striatal prediction-error signals following acute stress in healthy females. Biol Psychiatry 3223:31371-31379. CrossRef Medline

Watson D, Clark LA, Tellegen A (1988) Development and validation of brief measures of positive and negative affect: the PANAS scales. J Pers Soc Psychol 54:1063-1070. CrossRef Medline 